\title{
Method For Quantifying VAlue of StORAge Toward REACHING 100\% RENEWABLE ELECTRICITY
}

PREPRINT

\author{
Rhonda Bailey \\ RB RE Consulting LLC \\ San Ramon, CA USA \\ rhonda@rbreconsulting.com
}

\author{
P. A. Sánchez-Pérez \\ University of California, Merced \\ Merced, CA, USA \\ pesapsanchez@gmail.com

\section{S. Kurtz} \\ University of California, Merced \\ Merced, CA, USA \\ skurtz@ucmerced.edu
}

\author{
Zabir Mahmud \\ University of California, Merced \\ Merced, CA, USA \\ zmahmud@ucmerced. edu
}

June 5, 2020

\begin{abstract}
Optimization models can be quite powerful in identifying a pathway to lowest cost zero-carbon energy systems. However, it is less obvious how to invert the models to calculate the target cost and duration of storage needed for that storage to be a significant solution. Storage is a dispatchable and flexible resource with the ability to perform many functions of grid support, further complicating the analysis. This paper complements existing papers by presenting an academic study of a simplified energy system, demonstrating a method for quantifying cost and duration targets for storage. The simplified analysis also helps to gain intuition about the synergistic relationship between storage and solar energy in a location like the state of California.
\end{abstract}

Keywords: storage, renewable energy penetration, cost targets, duration targets

\section{Introduction}

As the world explores how to eliminate carbon emissions while providing low-cost, reliable energy, many different approaches have been proposed. Solutions that have been proposed using geographically available resources [1, 2] include pumped hydro storage [3, 4], expanded transmission, [5, 6] and other approaches [7]. While the solutions may vary, all agree that some form of storage will be needed to reach $100 \%$ renewable electricity.

There are many storage technologies being developed. Each technology is characterized by unique advantages and disadvantages with only pumped hydro storage currently deployed at a significant scale. Some storage technologies have high charge-discharge cycle efficiencies. Some storage technologies can store energy for months without significant reduction in the charge-discharge cycle efficiency. Pumped hydro has demonstrated ability to meet both cost and efficiency targets in some locations and is now being considered in a wider range of locations. A common need amongst the many approaches to storage is to reduce cost while retaining an acceptable efficiency. Though it is obvious that lower cost will increase adoption of storage, cost targets to drive wider adoption are not currently well-defined. As new storage technologies are evaluated, it is useful to establish a methodology for how to quantify 1) the needed cost targets (which depends on the efficiency) and 2) the needed number of hours of storage.

In this paper we complete an academic study to demonstrate a methodology using a simplified approach. We start by defining the geographical area (the U.S. state of California), the modeling software (RESOLVE), and a single day of weather and load data. We report modeling results as a function of the storage cost and analyze these in the context of the total cost of implementing a zero-carbon grid, the extent to which storage is used in that implementation, and the 
evolution of the number of hours of storage needed during that implementation. We then compare those results for a hypothetical case, showing how results would be different if California had a low-cost low-carbon alternative to storage. We conclude with an evaluation of how this methodology may be applied in practical situations and note the synergies between storage and solar in an area like California, which has abundant solar resource.

\section{Definition of academic approach}

\subsection{Geographical area selection}

The state of California has set a target of reaching zero- carbon energy by $2045[8]$ and has invested substantial effort in defining what would be needed to reach such a goal. The California Energy Commission (CEC) uses a Reference System Portfolio (RSP) [9, 10] as a realistic baseline, incorporating existing plants and plans for both new construction and retirements, while reaching the state's goals for renewable electricity and greenhouse gas emissions reductions by 2045. California was reported by the Energy Information Agency (EIA) to have generated 20\% of electricity from solar in 2019 [11]. This relatively high level of solar penetration, coupled with California's plans to achieve zero-carbon, make it an excellent testbed for this study.

\subsection{Modeling software and data inputs}

We have selected RESOLVE [9] as the modeling software because it is the software that the CEC used for defining the RSP. RESOLVE was written in Python by E3 with support from the CEC. It is an optimization program that can use a number of commercial solvers and calculates the minimum cost for building out and operating the electricity grid in California subject to greenhouse gas emission constraints. RESOLVE typically is run for 37 representative days, matching the generation with the load at every hour, with each day weighted so that the collective weighting reflects statistics for the whole year. RESOLVE also is typically run for several years, incorporating the expected changes in load and generation from 2020 through 2045. For this paper, to demonstrate the methodology and simplify the understanding of the results, optimizations were simplified to run for a single representative day in July, over the course of the 25-year span.

We use the input parameters defined in the RSP (making only the change of selecting a single day) as a starting point for our evaluation. The RSP is described in a companion paper in these proceedings [12]. We replace the RSP's default storage cost projections with a range of values to evaluate the effect of cost. We also explore the hypothetical situation of California having unlimited low-cost low-carbon generation capability available as an alternative to storage by removing the build-out capacity limit for wind generation in Solano County, California (setting the "capacity_limited" flag to 0 in the file resources.tab and deleting 8 Solano_Wind input lines from the file capacity_limits.tab).

RESOLVE models the cost of the storage as an annual cost, with an input for the capital cost (distributed over the life of the system) and fixed operations and maintenance (O\&M) cost (with variable O\&M costs distributed over all years equally). For these simulations, when the storage cost was varied, the same cost was used for systems installed in all years and the O\&M cost was fixed at one-tenth of the capital cost (captured in variables energy_storage_cost_dollars_per_kwh_yr and new_energy_capacity_fixed_o_and_m_dollars_per_kwh_yr in the input file resource_vintage_storage_params.tab). RESOLVE separates out different types of storage resources for different technologies including pumped hydro, flow and lithium ion technologies. For this study, we only varied the costs of the lithium ion technology resources 1-4 in the CAISO region. The assumed efficiency of the CAISO lithium ion resources was fixed at $92.2 \%$ for each of the charging and discharging processes for all years. The minimum duration hours for these four resources was retained at the RSP value of one hour.

We recognize that by fixing cost and efficiency for lithium ion resources throughout the 25-year range of simulations, we are not capturing the expected "real-life" cost and efficiency improvement as technologies mature. In a refined implementation of the method, we would define a learning curve, then vary the input cost parameters to match the associated cost reduction with time. In the refined case, we would plot the results as a function of the slope of the learning curve, but for this demonstration it is easier to reference the assumed fixed cost. By understanding the effect of varying the cost inputs, we can start to extract targets for cost and duration needed for storage to disrupt the energy landscape in each year of the simulation.

\subsection{Selection of one day}

In a further simplification, we have selected one of the 37 days for this study in order to facilitate an understanding of the methodology. We selected a day in July because the peak electricity demand typically occurs in July and because 
JUNE 5, 2020

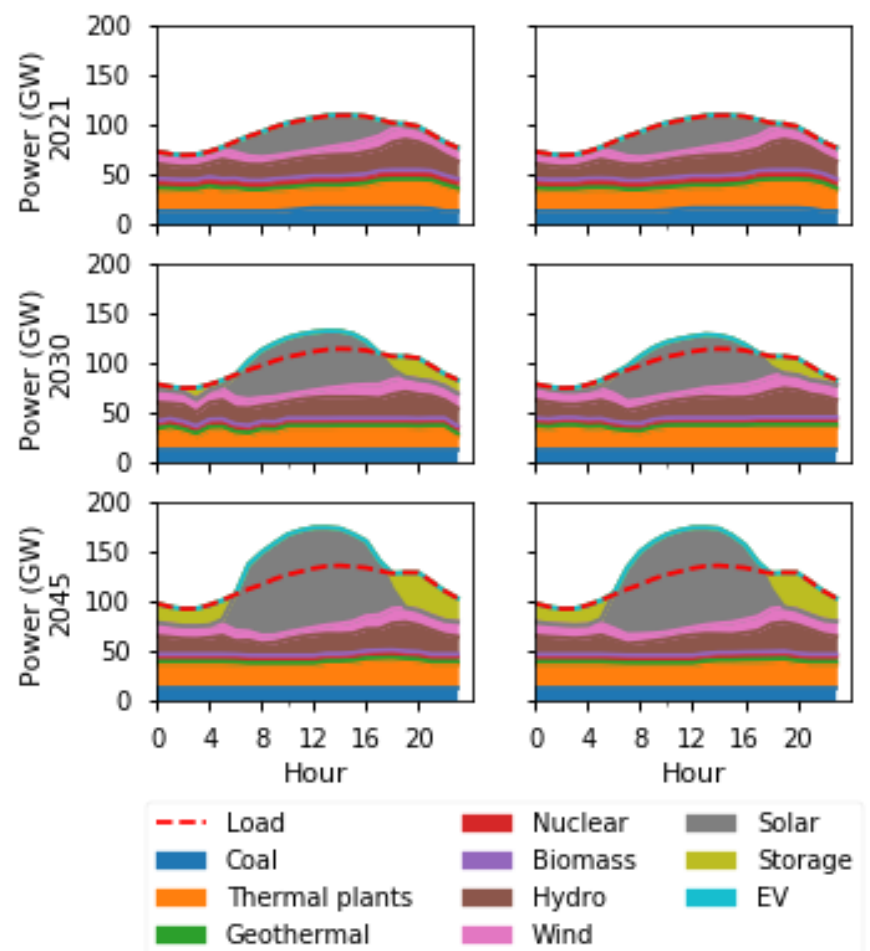

Figure 1: Modeled electricity generation in CA for each type of generation in 2021, 2030, and 2045. Storage capital costs are $\$ 1 / \mathrm{kWh} / \mathrm{y}$ on left and $\$ 12 / \mathrm{kWh} / \mathrm{y}$ on right.

the weather in California in July tends to be stable. The selected day is day 13 in the RESOLVE software. The day was modeled on actual data from July 17, 2007. This gives a well-defined day for demonstrating the methodology.

We emphasize that the results reported based on one day are not intended to describe the total situation in California. Insights gained into the results for this day will not provide information about the challenge of meeting electricity demand with renewable energy in California at other times of year. In particular, we note that in the winter, California may experience multiple days with very little sunshine, and, if heat pumps become widely adopted, the peak electricity load could shift to wintertime in California, suggesting that seasonal storage will become essential. Rather, the analysis of one day enables an in- depth presentation of the method as a basis for further study.

\section{Results and discussion}

\subsection{Electricity generation by technology}

The electricity generation by technology as a function of hour of day for the selected day simulated in 2021 (essentially today's mix of electricity-generating capacity), 2030, and 2045 (meeting California's targets) is shown in Fig. 11 The left-hand graphs show low cost storage $(\$ 1 / \mathrm{kWh} / \mathrm{y})$ and the right-hand graphs show high cost storage $(\$ 12 / \mathrm{kWh} / \mathrm{y})$.

As shown in Fig. 1. generation that exceeds the load during the day (area of the curve above the red dotted line) is used to charge the storage, then the storage is discharged (green area) during the night. As expected, to meet the 2045 zero-carbon target, the amount of storage is increased substantially by 2045. However, the build out of storage changes only slightly in these optimizations despite the large (factor of 12) difference in cost of storage assumed for the two cases.

To provide the optimization an alternative way to meet the emissions target, we allowed additional wind plants to be installed. We selected the Solano wind resource that is included in the RSP, but normally limited in build out to 0 MW in 2021 and 542 MW thereafter (capacity_limit_mw in input file capacity_limits.tab). When the capacity limit lines were removed for this resource, the optimization selected to build more than $30 \mathrm{GW}$ of Solano Wind by 2045 . The results are shown in Fig. 22, showing that wind electricity can now be used through most of the night. Less storage is 
JUNE 5, 2020

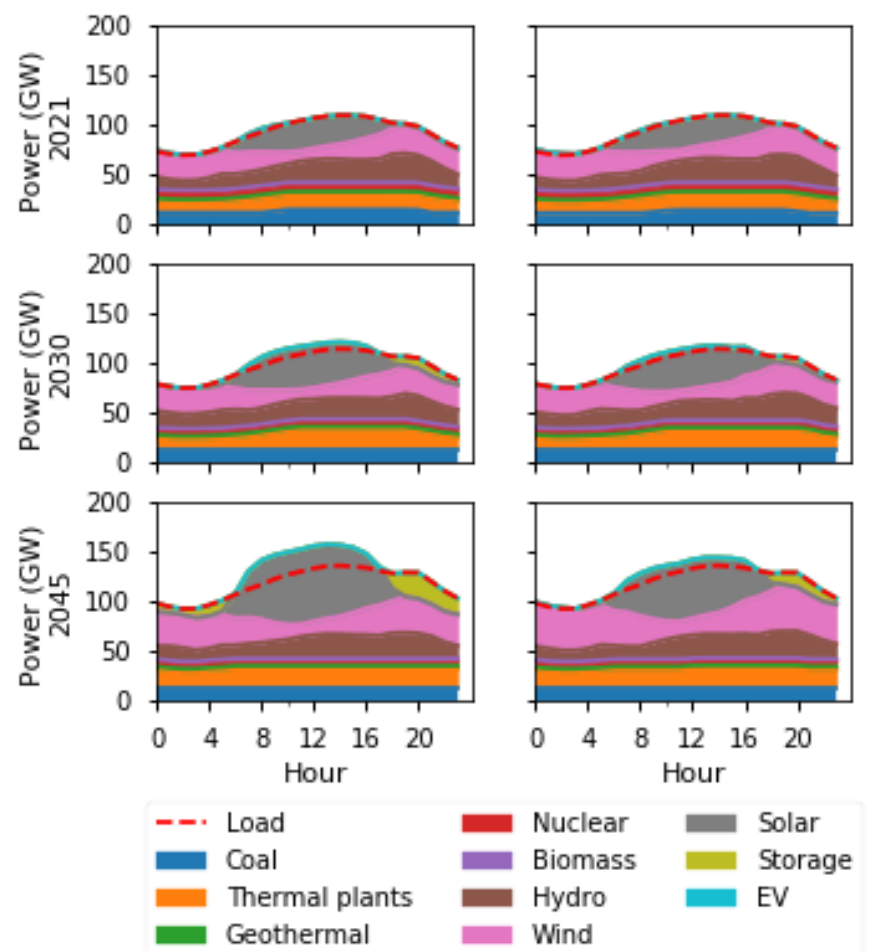

Figure 2: Same data as in Fig. 1 but providing a low-cost alternative to storage by allowing the Solano Wind resource build out to be unlimited.

now built out as compared to Fig 1 and the cost difference now has visible impact on the storage build out by 2045 . Storage build out is further quantified in the next section.

\section{Metrics for quantifying value of storage}

We propose that there are two key metrics for quantifying the overall value of storage: 1) overall cost of the system and 2) extent to which the optimization selects the storage option (the needed reduced cost that will result in storage being selected as the preferred solution).

We first explore the total cost of implementing the optimized modeled scenarios in 2045, as shown in Fig. 3, left axis. When the reference scenario was used, an increase in cost of the storage from $\$ 1 / \mathrm{kWh} / \mathrm{y}$ to $\$ 12 / \mathrm{kWh} / \mathrm{y}$ increased the total system cost by $11 \%$. When wind was offered as an alternative source of electricity during the night, not only was the total cost reduced, but the same increase in storage cost resulted in $<5 \%$ increase in system cost.

We plot the modeled operational capacity for all of the storage resources in 2045 as a function of input battery cost in Fig. 3. right axis. We see that for the RSP (black dots), storage is the only generation category that can be built to provide nighttime generation and meet the greenhouse gas targets, so the total capacity of the storage resources in Fig. 3 does not change significantly as cost varies. However, we see that the total system cost does decrease as storage cost is reduced. In this case, the primary benefit of lower cost storage is in reduced total system cost rather than in more storage capacity being built out. Conversely, considering the case when an alternative low-cost renewable (wind) is not limited, the total system cost benefits less from a reduced cost of storage, but we see a much greater change in the capacity of storage adopted (red dots) as the storage changes in cost.

In the RSP case, reducing the cost by about $20 \%$ (from $\$ 12 / \mathrm{kWh} / \mathrm{y}$ to $\$ 10 / \mathrm{kWh} / \mathrm{y}$ ) shows more benefit in reduced total system cost than reducing a $\$ 2 / \mathrm{kWh} / \mathrm{y}$ cost by $50 \%$ to $\$ 1 / \mathrm{kWh} / \mathrm{y}$, suggesting that a target price of $\$ 10 / \mathrm{kWh} / \mathrm{y}$ would be useful, though a lower cost would provide even more benefit, so identifying $\$ 10 / \mathrm{kWh} / \mathrm{y}$ may be considered a bit arbitrary. In the case where storage is competing with an alternative, the cost target for storage to be a valuable part of the solution may be taken as about $\$ 2 / \mathrm{kWh} / \mathrm{y}$ because reduction of the cost below $\$ 2 / \mathrm{kWh} / \mathrm{y}$ had very little effect on either the total cost or the amount of storage adopted. It's not a surprise that the needed cost for storage to be useful is 
JUNE 5, 2020

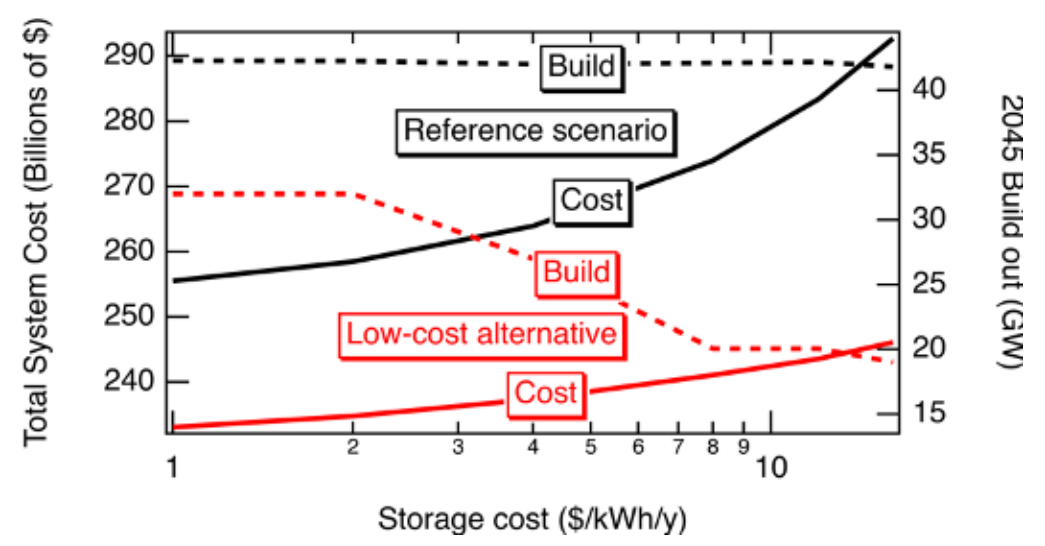

Figure 3: Total system cost (left axis) and storage capacity by 2045 (right axis) as a function of the input storage capital cost, modeled for the reference scenario in black and with an alternative low-cost solution (wind) in red. Figs. 1 and 2 show generation profiles for the optimizations with the minimum $(\$ 1 / \mathrm{kWh} / \mathrm{y})$ and maximum $(\$ 12 / \mathrm{kWh} / \mathrm{y})$ costs shown here.

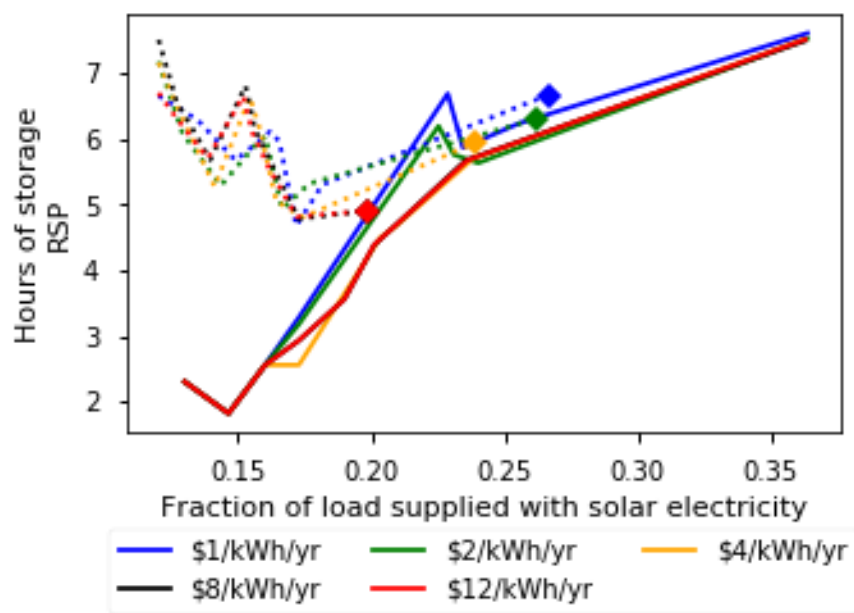

Figure 4: Storage duration as a function of the solar penetration for the RSP (solid) and for the RSP with low-cost alternative (dotted).

lower when there is a low-cost alternative available. This underscores the importance of appropriately identifying the lowest cost alternative when quantifying the target cost for storage.

\subsection{Value of storage as function of PV penetration}

To fully understand the role that storage plays, both the power capacity and duration must be analyzed. Our hypothesis is that both cost and other renewable penetration will impact the duration of storage built. We calculate the number of hours of storage that is used as a function of solar penetration as shown in Fig. 4 with solid lines for the 1-day RSP scenarios and with dotted lines terminated with diamonds for the 1-day low-cost alternative scenarios. The number of hours of storage duration can be calculated in many ways. We have defined it as the total discharged energy for all the deployed storage in the system divided by the maximum hourly discharged power from storage for the system.

In Fig. 4, we see that for the RSP, the hours of storage used increases with solar penetration as expected, approaching the number of hours of electricity needed at night. On the other hand, the hours of storage change very little with storage cost (see the rainbow of colors). The multiple lines are essentially on top of each other for low (below 17\%) and high (above 25\%) levels of solar penetration. The storage duration only diverges, with the lowest cost storage being built out slightly more, when the fraction of electricity from solar falls between about $17 \%$ and $25 \%$. Surprisingly, for the portfolio with a low-cost alternative, the hours of storage built out is relatively independent of both solar penetration and cost. 
JUNE 5, 2020

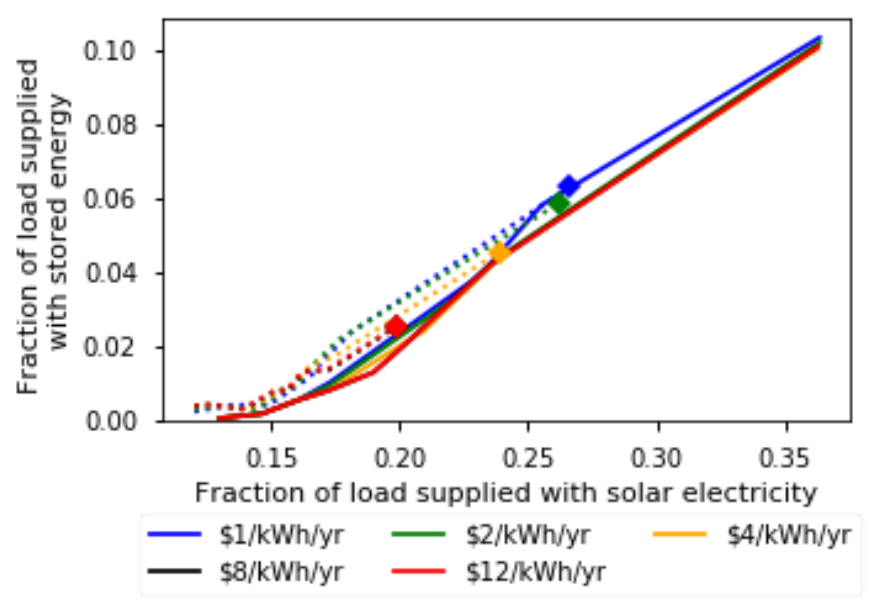

Figure 5: Fraction of electricity supplied by storage as a function of the fraction from solar for the RSP (solid) and for the RSP without wind constraint (dotted, with final data point highlighted with a dot).

We also evaluate the fraction of load met by storage as a function of solar penetration in Fig. 55 Once again, the curves corresponding to different storage cost for the 1-day RSP scenarios are shown as solid lines and the curves for the 1-day low-cost alternative scenarios are shown as dotted terminated with diamonds. In this case at lower solar penetration, the difference between the RSP and the RSP with low-cost alternative is small, indicating that though Fig. 4 showed shorter duration storage adoption for the RSP than for the case with a low-cost alternative, similar energy load is supplied with stored energy. Another significant difference between the scenarios in Figs. 4 and 5 is that the PV capacity does not get built out past $25 \%$ penetration when a low-cost wind alternative is present, highlighted by the placement of the terminating diamonds on both graphs.

The relationship between the storage and solar shown in Fig. 5 is strikingly linear. We anticipate that this slope or the intercept would change if we repeated the study for a day with different load shape. This simplistic study allows us to gain some intuition about the roles of solar and storage. Perhaps most surprising about this figure is that the relationship between fraction of electricity from solar and fraction from storage is similar for both the situation when wind is abundantly available and not.

In both Fig. 4 and Fig. 5 we confirm that the capacity of storage that is needed increases as the penetration of renewable electricity increases. For the RPS, both the hours and load supplied grow consistently with PV penetration. When a low-cost alternative with 24-hour generation capability is present, although the hours of storage needed are not well-correlated to PV penetration, the load supplied by storage still shows growth correlated with PV penetration.

\subsection{Value of storage depends on multiple factors}

We anticipate that the role of storage (primarily characterized by number of hours of storage needed) will change as the penetration of renewable electricity increases, as shown schematically in Fig. 6 .

Comparison of Fig. 1 and 2 shows how the value of storage depends on what other options are available. For simplicity and to allow for demonstration of the analysis methods, we only allowed wind as an alternative to storage in this current work. However, in addition to wind, we anticipate that demand management and a number of other approaches will affect the extent to which low-cost storage is useful toward balancing supply and demand at a low overall system cost. We see that in the near term for the RPS optimization, storage durations of 2 hours may meet much of the needs, but that as we move toward high penetration, longer duration storage will be needed. This one-day simulation neglects the variability of weather through the year and the RESOLVE code is not currently able to consider situations in which storage will need to carry electricity through cloudy days.

To extend this academic study to inform setting of targets for storage, more than one day's weather must be considered. This will require both modification of the software to simulate multiple days when wind and solar are not abundant and to vary the weather to identify risk of running out of electricity on some days.

The more complete study with inclusion of various weather scenarios will require an additional metric: in addition to those discussed above, the risk of running out of electricity on cloudy days is a key metric. Today, the system's reserve margin is likely to be critical on a hot day in the summer. In these cases, peaker plants have historically been used to 
JUNE 5, 2020

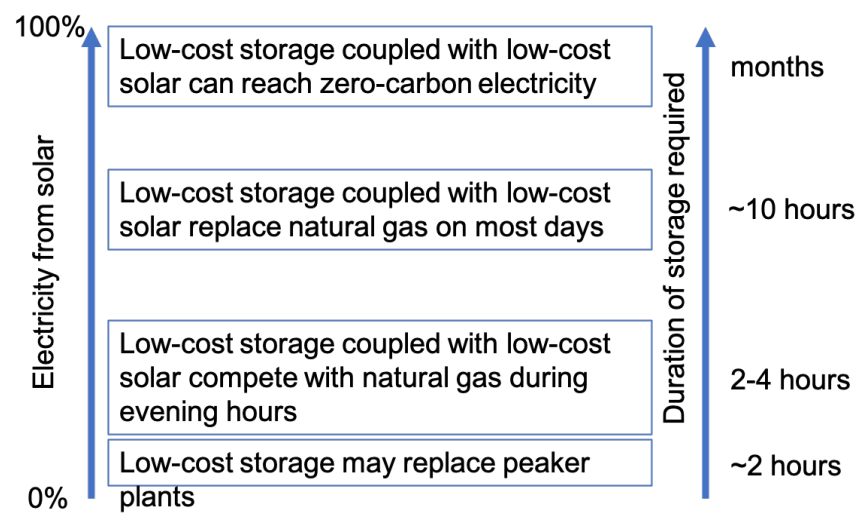

Figure 6: Schematic describing how the anticipated optimal duration of storage will increase as the penetration of solar electricity increases.

reduce the risk of under-generation. In a zero-carbon grid that uses mostly solar and wind, the risk will come on days when the sun isn't shining and the wind isn't blowing. Larger storage capacity and especially storage capacity that can provide longer duration of discharge may be critical in those cases.

\section{Conclusions}

We have shown that the cost target for storage can be derived using an optimization model like RESOLVE by varying storage cost and plotting both the total system cost and the extent to which storage is used as a function of the assumed storage cost. From this graph, we can identify the storage cost that will result in the described storage being chosen over a competing solution and to quantify the reduction in total system cost for a given storage cost target. However, we also show that the choice of constraints used in the optimization model can lead to different results.

The number of hours of storage that are needed is shown to be surprisingly independent of storage cost. It is also shown to behave differently compared to PV penetration depending on the other parameters in the system definition (whether a low-cost alternative is available to the optimization). However, the load supplied by storage is shown to strongly correlate to penetration of the solar electricity, regardless of cost or other system details.

Among other parameters, these metrics depend on 1) the location (which determines the available renewable energy resources), 2) the weather (especially if there are prolonged times of year when neither solar nor wind energy is available), 3) load profiles, and 4) the penetration level of the solar and wind electricity. It is likely that $100 \%$ renewable electricity systems around the world will end up with different requirements. In some locations, seasonal storage (storage of large quantities of energy for months) may not be needed at all. In other locations, seasonal storage will be required. As companies race to provide storage technologies with the needed attributes, it is essential that we have robust methods for comparing different technologies in different regions.

Due to the simplifications made during this analysis (using 1 day of results, varying only lithium ion storage costs in CAISO, and choosing wind as a low cost alternative with a 24-hour generation capability to build out unconstrained) the results presented throughout the paper are not meant to be viewed as representative of final real-world results. Rather, they are results for simplified cases invented to show the simulation and methods used to analyze the value of storage in a low-carbon future. Further work will explore more broadly applicable cases.

\section{Acknowledgment}

The authors thank University of California Merced under-graduate group Michele Campbell, Joseph Bello, and Jeremiah Tower for their useful work in developing the python scripts. We also thank Doug Condon for useful comments.

\section{References}

[1] A. Mileva J. Johnston, J.H. Nelson, D.M. Kammen, "Power system balancing for deep decarbonization of the electricity sector," Appl. Energy, vol. 162, pp. 1001-1009, 2016. 
[2] A. Aghahosseini, D. Bogdanov and C. Breyer, "A techno-economic study of an entirely renewable energy-based power supply for North America for 2030 conditions," Energies, vol. 10, (8), pp. 1171, 2017.

[3] M.Z. Jacobson, M.A. Delucchi, "Providing all global energy with wind, water, and solar power, Part I: Technologies, energy resources, quantities and areas of infrastructure, and materials," Energy Policy, vol. 39, (3), pp. 1154-1169, 2011.

[4] A. Blakers, B. Lu, M. Stocks, "100\% renewable electricity in Australia," Energy, vol. 133, pp. 471-482, 2017.

[5] D. Z. Fitiwi et al, "Finding a representative network losses model for large-scale transmission expansion planning with renewable energy sources," Energy, vol. 101, pp. 343-358, 2016.

[6] T. Brown et al, "Synergies of sector coupling and transmission reinforcement in a cost-optimised, highly renewable European energy system," Energy, vol. 160, pp. 720-739, 2018.

[7] J. P. Barton and D. G. Infield, "Energy storage and its use with intermittent renewable energy," in IEEE Transactions on Energy Conversion, vol. 19, no. 2, pp. 441-448, June 2004, doi: 10.1109/TEC.2003.822305

[8] California LEGISLATIVE INFORMATION, "SB-100 California Renewables Portfolio Standard Program: emissions of greenhouse gases", Sep. 2018. Accessed: Jun. 01, 2020. [Online].

[9] California Public Utility Commission, "2019-2020 Electric Resource Portfolios to inform Integrated Resource Plans and Transmission Planning,” 331772681, Apr. 2020. Accessed: Jun. 01, 2020. [Online]. Available: https: //bit.ly/3eGqGRg

[10] California Public Utility Commission, "Inputs \& Assumptions: 2019- 2020 Integrated Resource Planning," Feb. 2020. Accessed: Jun. 01, 2020. [Online]. Available: https://bit.1y/2XUjudi

[11] U.S. Energy Information Administration, “ELECTRICITY DATA BROWSER”, Mar. 2020. Accessed: Jun. 01, 2020. [Online].

[12] P. A. Sanchez-Perez and S. Kurtz, "California's Vision for Reaching Zero-Carbon Emissions", 47th PVSC proceedings, in press. 